\title{
Applications of Bicrystallography: Revealing Generic Similarities in Coincidence Site Lattice Boundaries of all Holohedral Cubic Materials and Facilitating the Design of 3D Printed Models of such Grain Boundaries
}

\author{
Peter Moeck ${ }^{1}$, Andrew Maas ${ }^{1}$, Jennifer Stone-Sundberg ${ }^{1,2}$, Bryant York ${ }^{3}$, Trevor Snyder ${ }^{1,4}$, Werner \\ Kaminsky $^{5}$, and Nigel Browning ${ }^{6}$ \\ 1. Nano-Crystallography Group, Department of Physics, Portland State University, Portland, OR 97207-0751 \\ 2. Crystal Solutions, LLC, Portland, OR 97205 \\ 3. Department of Computer Science, Portland State University, Portland, OR 97207-0751 \\ 4. 3D Systems Corporation, Wilsonville, OR 97070 \\ 5. Department of Chemistry, University of Washington, Seattle, WA 98195 \\ 6. Chemical and Materials Sciences Division, Pacific Northwest National Laboratory, Richland, WA 99352
}

The application of bicrystallography [1-3] makes structural units [4-6] and dislocations [7] superfluous as descriptors of grain boundaries. All there is around an ideal (un-relaxed) coincidence site lattice (CSL) grain boundary are predicable atomic positions with certain black-white symmetries. Free energy minimization driven relaxations of such a hypothetical grain boundary structure may lead to the breaking of some or all of the black-white symmetries, just as monochrome space group symmetries may be broken locally in the two real single crystals that make up the bicrystal. Any relaxed (real) atomic position will, however, be very close to the predicted un-relaxed (ideal) atomic position as long as that position remains occupied. Grain boundary structures with nine degrees of freedom as predicted by bicrystallography in three dimensions (3D) are, therefore, ideal as atomic level starting structures for free energy minimization calculations! Bicrystallography in two dimensions (2D) allows for both visualizations of edge-on projections of CSL tilt boundaries [3] and the extension of the atomic column indexing procedure of ref. [8] to all experimental 2D images of such boundaries. In order to illustrate the predictive power of bicrystallography in 2D briefly, we reproduce below in Figure 1 migration related segments of translation averaged Z-STEM images of a $\Sigma$ 13a (510) tilt boundary in $\mathrm{SrTiO}_{3}$ in [001] projection from ref. [3] (with permission from the publisher).

A range of CSL [001] tilt boundaries in $\mathrm{CeO}_{2}$ were imaged with an aberration corrected Z-contrast Scanning Transmission Electron Microscope (Z-STEM) and striking visual similarities of their (projected) structural units to those of (analogous orientation relationship) grain boundaries in face-center cubic metals, yttriastabilized zirconia, and $\mathrm{SrTiO}_{3}$ (Fig. 1) were recently noted in ref. [4]. There is, however, some arbitrariness in the choosing of structural units [7], despite their emergence in connection with free energy minimization calculation [5] and their identification as cores of grain boundary dislocations [6]. In essence, they are arrangements of atoms from both sides of a grain boundary with geometries that can be chosen "simply for convenience" [7]. The predictive power of structural units is, accordingly, limited to series of grain boundaries in the same material when only one of the five macroscopic degrees of freedom is varied [7]. For predictions of the structures of tilt boundaries on the basis of structural units, the parameter that varies in a series is most often the tilt angle, e.g. see ref. [4].

Our bicrystallography analysis, on the other hand, shows that these structural similarities are simply byproducts of the edge-on projection of the black-white layer groups of the bicrystals in these materials [3]. In short, whenever Bärnighausen trees [9] reveal structural relations (i.e. similarities) between the space groups of different materials, there will (per bicrystallography) also be predicable structural similarities in their analogous orientation relationship grain boundaries. The bicrystal layer groups of all CSL [001] tilt boundaries in the above mentioned (cubic holohedral) materials project along [001] to black-white frieze symmetries $\mu 11 m^{\prime}$ (Figs. 1a and 1b), $\mu 11 g^{\prime}$ (Figs. 1c and 1d), and $\mu 111^{\prime}$ [3] so that we can use simple sketches 
of 2D projections of CSL [001] tilt boundaries to reveal the above mentioned structural similarities visually. Such sketches have been produced with the popular drawing program GIMP on the basis of both our 2D bicrystallography procedure [3] and supporting MATLAB code outputs that generate [001] projections of the structure of the single crystal components of cubic holohedral bicrystals [10].

We also produced 3D printed hand-held models of CSL [001] tilt and twist boundaries in some of the above mentioned materials in order to demonstrate their generic structural similarities at the atomic level. The Cif2VRML program [11] was used for the design of these models. This program reads atomic coordinates of grain boundary structures in the very well documented Crystallographic Information File (CIF) format [12] and converts them directly into 3D print file formats. In order to provide a concise structure description in the corresponding CIFs, the bicrystal layer group symmetry of a grain boundary can stand in for the space group symmetry of the component crystals and the CSL parameters can stand in for these crystals' unit cell parameters. In the CIFs that encode the structure of the highest symmetric grain boundary models, only one eighth of the atoms that make up the 3D printed models (i.e. the content of the "asymmetric black-white unit" of the CSL unit cell) need then be included explicitly. Due to the inherent conciseness of these kinds of "bicrystal layer group/CSL parameter" CIFs, we propose to make them the new standard for the encoding of atomic level starting structures for free energy minimization calculations of grain boundaries [13].

[1] R. C. Pond and W. Bollmann, Proc. Royal Soc. London A 292 (1979) 449.

[2] R. C. Pond and D. S. Vlachavas, Proc. Royal Soc. London A 386 (1983) 95.

[3] P. Moeck et al, Cryst. Res. Technol. 49 (2014) 708.

[4] W. Tong et al, Acta Mater. 61 (2013) 3392.

[5] A. P. Sutton and V. Vitek, Phil. Trans. Royal Soc. London A 309 (1983) 1, 37, 55.

[6] N. D. Browning et al, In: Modeling Nanoscale Imaging in Electron Microscopy, eds. T. Vogt et al, (Springer, 2012).

[7] L. Priester, Grain Boundaries From Theory to Engineering, (Springer, 2013).

[8] X. Sang et al, Microsc. Microanal. 20 (2014) 1764.

[9] U. Müller, Symmetry Relations between Crystal Structures, (Oxford University Press, 2013).

[10] A. Maas, Capstone project, http://nanocrystallography.research.pdx.edu/media/andrew-capstone.pdf.

[11] W. Kaminsky et al, Powder Diffraction 29 (2014) S42. The program is freely downloadable at http://cad4.cpac.washington.edu/Cif2VRMLHome/Cif2VRML.htm.

[12] http://www.iucr.org/resources/cif

[13] The authors acknowledge support from the National Science Foundation (grant EEC-1242197), 3D

Systems Corporation, and the U.S. National Committee for Crystallography.
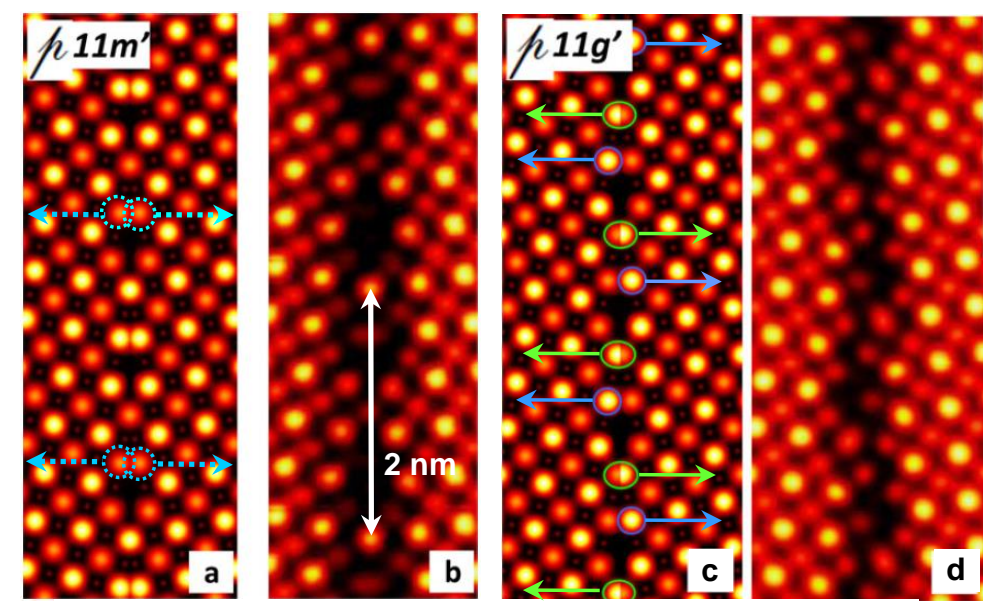

modified after P. Moeck et al., Cryst. Res. Technol. 49 (2014) 708
Figure 1. Edge-on projections of migration related segments of a $\Sigma 13$ a (510) tilt boundary in $\mathrm{SrTiO}_{3}$ (after ref. [3]). a and $\mathbf{c}$, as predicted by bicrystallography in $2 \mathrm{D}$, $\mathbf{b}$ and $\mathbf{d}$ as experimentally observed by Z-STEM imaging and translation averaging. The marked mixed $\mathrm{Ti} / \mathrm{O}$ and $\mathrm{Sr}$ columns in a and $\mathbf{c}$ must be in energetically unfavorable positions because their counterparts are (in essence) not occupied in $\mathbf{b}$ and $\mathbf{d}$. Note that the black-white symmetries in the bicrystallography predictions (a,c) are not broken (at least on average) by atomic level free energy minimization effects that show up in the experiments $(\mathbf{b}, \mathbf{d})$. 